\title{
Closed Total Talar Dislocation with Associated Tarsal Fractures: Case Report and a Review of the Literature
}

\section{Andrew Chia Chen Chou $^{1, *}$ and Mohan Tiruchittampalam ${ }^{2}$}

${ }^{1}$ Department of Orthopaedic Surgery, Singapore General Hospital, The Academia, 20 College Road, Singapore

${ }^{2}$ Senior Consultant, Department of Accident and Emergency, Changi General Hospital 2 Simei Street 3, Singapore

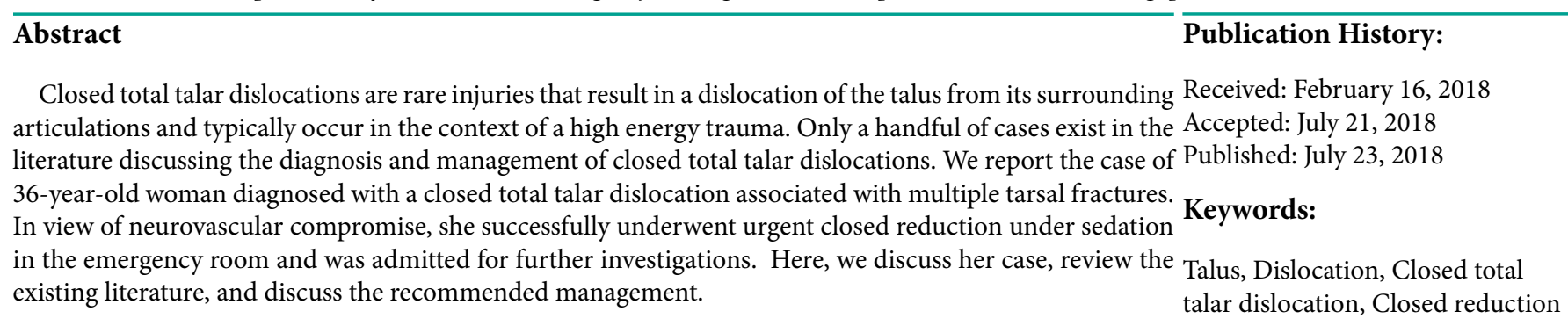

\section{Introduction \& Background}

As over five million ankle injuries occur annually in the United States alone, foot and ankle injuries account for up to $30 \%$ of emergency room visits and up to $12 \%$ of traumatic injuries [1-3]. While most patients do not require imaging and can be treated on the outpatient basis, emergency physicians need to be capable of recognizing complex cases and the need for urgent orthopaedic consultation $[1,3]$. While most ankle trauma is not limb-threatening, if associated with neurovascular deficits or open injuries, ankle dislocations and fractures need to be recognized and treated urgently $[1,2]$.

A total talar dislocation is defined as a dislocation of the talus from its surrounding articulations - talonavicular, subtalar, and tibiotalar - and is typically secondary to a high energy trauma [2,4-8]. While talar dislocations themselves are rare, the vast majority of cases are open dislocations and only a handful of closed talar dislocations are documented $[4,7,8]$. As the vascularity of the talus is delicate and reliant on the surrounding soft tissue attachments, prompt closed reduction paying respect to the surrounding soft tissue is critical to prevent complications such as infection, avascular necrosis, and posttraumatic osteoarthritis $[4,6,7,9]$. As such dislocations are rare, the standard of care for closed talar dislocations is not well established $[4,6,7]$. Here, we report the case of 36-year-old female who sustained a close total talar dislocation with associated tarsal fractures, review the existing literature, and discuss the recommended management.

\section{Case Report}

\section{History of presenting illness}

Miss $\mathrm{P}$ was 36-year-old female Malay teacher with no significant past medical history and no history of smoking or drinking. While wearing 2-inch heels, she missed a step while walking down stairs, slipped, and fell, landing on her heel. Subsequently, she developed right heel pain and swelling with inability to weight bear on her right lower limb. She sustained no other injuries and was otherwise systemically well.

\section{Physical examination}

On examination, Miss $\mathrm{P}$ was alert, comfortable, and not in obvious distress. The right medial malleolus was tender to palpation with mild swelling, but no tenderness over the base of foot and no open wounds were noted. Her right ankle's range of motion was severely limited by pain and could not be well assessed. The dorsalis pedis pulse was not palpable, but was detectable weakly with a Doppler probe. The posterior tibial pulse was well felt. Capillary refill time was brisk and sensation in the toes was grossly intact.

\section{Imaging}

The initial right ankle anteroposterior and lateral radiographs and right foot oblique and dorsal-plantar radiographs are shown in Figure 1. A complex dislocation of the proximal intertarsal joints is seen with the talus dislocated laterally relative to the calcaneum. Additionally, dislocation of the talonavicular and calcaneocuboid joints is also seen. An avulsed fracture fragment can be seen between the talus and calcaneum in the dorsal-plantar view of the foot. Soft tissue swelling can be also be appreciated over the lateral malleolus.
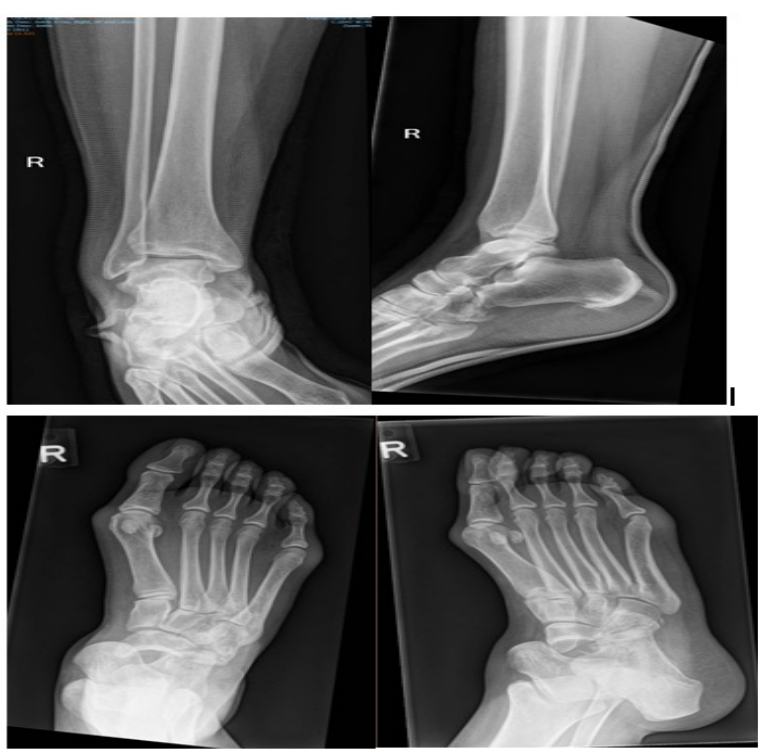

Figure 1: Initial right ankle and foot radiographs taken on admission.

${ }^{*}$ Curresponding Author: Dr. Andrew Chou, Department of Orthopaedic Surgery, Singapore General Hospital The Academia, 20 College Road, Singapore 169856; Email: andrew.chou@alumni.stanford.edu

Citation: Chou A, Tiruchittampalam M (2018) Closed Total Talar Dislocation with Associated Tarsal Fractures: Case Report and a Review of the Literature. Int $J$ Surg Surgical Porced 3: 137. https://doi.org/10.15344/2456-4443/2018/137

Copyright: @ 2018 Chou et al. This is an open-access article distributed under the terms of the Creative Commons Attribution License, which permits unrestricted use, distribution, and reproduction in any medium, provided the original author and source are credited. 
Citation: Chou A, Tiruchittampalam M (2018) Closed Total Talar Dislocation with Associated Tarsal Fractures: Case Report and a Review of the Literature. Int J Surg Surgical Porced 3: 137. https://doi.org/10.15344/2456-4443/2018/137

Page 2 of 3

\section{Treatment}

In view of the poorly felt dorsalis pedis pulse and the complex foot dislocation seen on radiographs, the patient was promptly referred to the orthopaedic specialist on-call for review. After adequate analgesia and sedation, the patient successfully underwent manipulation and reduction of her right foot intertarsal dislocation. Post-reduction radiographs showed adequate reduction of the tarsal dislocation with congruent talonavicular, talocalcaneal, and calcaneocuboid joints and an intact ankle mortise and can be seen in Figure 2. Following the reduction, the dorsalis pedis pulse was still not well felt, although the Doppler signal was more apparent. Subsequently, she was admitted to the inpatient orthopaedic service for further imaging and limb circulation monitoring.

\section{Follow-up}

Computed tomography imaging of her right foot showed a comminuted intra-articular fracture of the anterior calcaneus with superior displacement of the distal fracture fragments and articular step-off. Additionally, a comminuted intra-articular fracture of the cuboid near the calcaneal-cuboid articulation with inferior displacement of the fracture fragments and articular step-off was seen. A small comminuted fracture of the sustenaculum tali extending intra-articularly to the middle subtalar joint was noted. Images from the CT foot are shown in Figure 3. In view of her CT findings, the patient was counselled for the possibility of shortening of the bone column and advised to avoid weight bearing on her right foot by ambulating crutches until her follow-up with an orthopaedic foot and
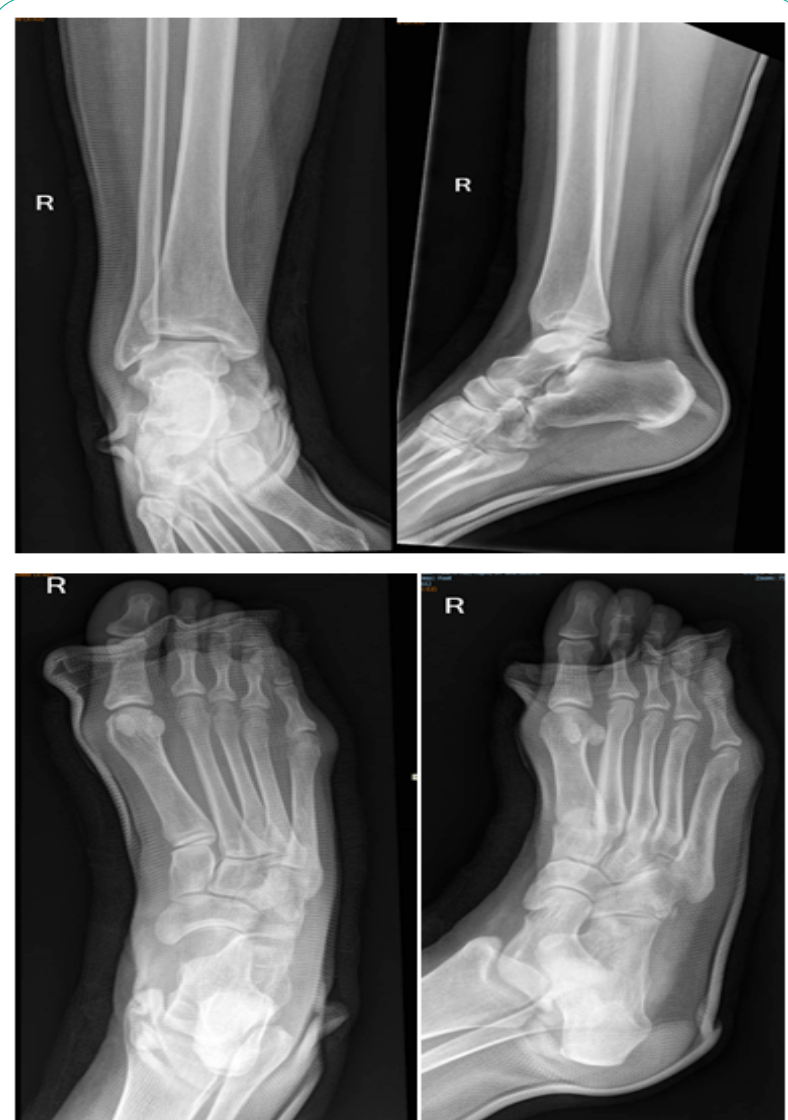

Figure 2: Post-reduction radiographs of the right foot and ankle. ankle specialist on discharge. Subsequent follow-up radiographs done four weeks after discharge indicated interval healing of the cuboid and calcaneal fractures and are shown in Figure 4.
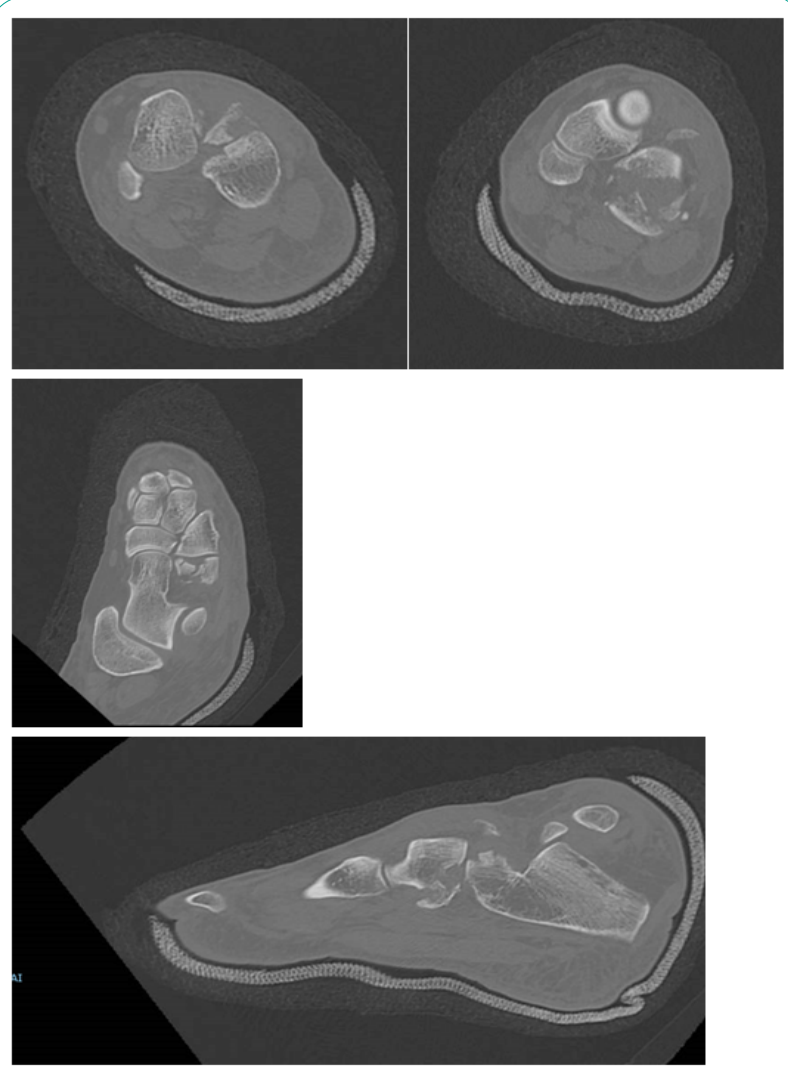

Figure 3: Post-reduction computed tomography of the right foot and ankle.
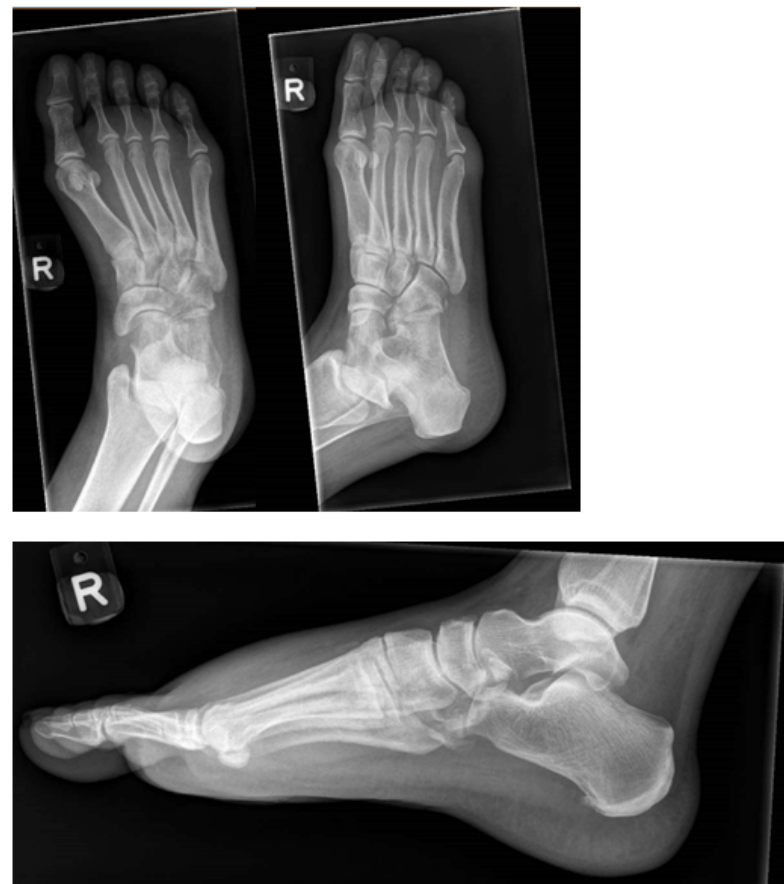

Figure 4: Post-discharge radiographs of the right foot. 
Citation: Chou A, Tiruchittampalam M (2018) Closed Total Talar Dislocation with Associated Tarsal Fractures: Case Report and a Review of the Literature. Int J Surg Surgical Porced 3: 137. https://doi.org/10.15344/2456-4443/2018/137

Page 3 of 3

\section{Discussion}

Total talar dislocations are rare and frequently open injuries that account for only $0.06 \%$ of dislocations and $2 \%$ of talar injuries in general $[6-8,10]$. A systematic review by Weston et. al. in 2015 showed that only 39 articles reporting a total of 86 cases of total talar dislocations were available in the literature [7]. Consequently, the standard of treatment and follow-up for total talar dislocations, particularly closed ones, is not well established $[4,7,8,10]$. The most common mechanism of injury was reported to be forced plantarflexion associated with forced eversion or inversion $[4,10]$. Associated foot and ankle fractures were found in 43 cases, in which 32 were specifically talar fractures [4,7]. In the context of total talar dislocations, open fractures and any neurovascular deficits warrant urgent orthopaedic referral [1-3].

Of the cases reviewed, only thirteen were noted to be closed and closed reduction was successful in only three of these cases [7]. While Ritsema previously advocated for open reduction as the choice of treatment for closed total talar dislocations, multiple case reports have shown good results with closed reduction [4,11-13]. Thus, early closed reduction should be attempted first for closed talar dislocations to avoid injury to surrounding soft tissue and delicate vasculature, with open reduction reserved for unsuccessful reductions $[4,8,10]$.

Due to the delicate blood supply of the talus, dislocations of the talus are often difficult to reduce and repair and frequently associated with complications, such as avascular necrosis, infection, and osteoarthritis $[4,7,9,10]$. The incidence of avascular necrosis was reported to be as high as $90 \%$, particularly in the first year after dislocation, and warrants a high index of suspicion during follow-up visits [4,7]. Serial radiographs performed over the months after reduction and close follow-up with an orthopaedic specialist are recommended to monitor for signs of avascular necrosis, with magnetic resonance imaging suggested as a more sensitive and specific adjunct $[4,9]$.

In comparison to the literature, the talar dislocation discussed in the case report differs in a few critical ways. First, despite talar dislocations typically occurring in the context of high energy forces with forced plantar flexion, the patient missed a step on the stairs while wearing high heels and landed on her heel. Furthermore, unlike most cases involving an open injury, the patient sustained a close talar dislocation, which occurs in fewer than $20 \%$ of documented talar dislocations. Lastly, prompt closed reduction was successful in restoring talar joint alignment, which has only been documented as successful in only a handful of cases. However, as recommended by the literature, early orthopaedic consultation and attempts at closed reduction were made promptly and the patient was discharged for follow-up with an orthopaedic specialist and repeat serial radiographs.

In summary, while foot and ankle injuries are common in the emergency room, closed talar dislocations with associated tarsal fractures are rare injuries that require high suspicion of injury and prompt management. In the event of neurovascular compromise, an urgent orthopaedic consultation and closed reduction should be attempted before considering surgical management. Due to the high risk of avascular necrosis and other complications, close follow-up with an orthopaedic specialist and serial radiographs or imaging are indicated on discharge.

\section{Competing Interests}

The authors declare that they have no competing interests

\section{References}

1. Birrer RB, Fani-Salek MH, Totten VY, Herman LM, Politi V et al. (1999) Managing ankle injuries in the emergency department. The Journal of emergency medicine 17: 651-660.

2. Hanlon DP (2010) Leg, ankle, and foot injuries. Emerg Med Clin North Am 28: $885-905$

3. Wedmore I, Young S, Franklin J (2015) Emergency department evaluation and management of foot and ankle pain. Emergency medicine clinics of North America 33: 363-396.

4. Heylen S, De Baets T, Verstraete P (2011) Closed total talus dislocation: a case report. Acta Orthop Belg 77: 838-842.

5. Hendin A, Rosenberg $\mathrm{H}$ (2017) The floating foot: a case of total talar dislocation. CJEM 19: 154-155

6. Melenevsky Y, Mackey RA, Abrahams RB, Thomson III NB (2015) Talar Fractures and Dislocations: A Radiologist's Guide to Timely Diagnosis and Classification. Radiographics 35: 765-779.

7. Weston JT, Liu X, Wandtke ME, Liu J, Ebraheim NE, et al. (2015) A systematic review of total dislocation of the talus. Orthop surg 7: 97-101.

8. Kumar YC, Reddy S, Golla DK, Ganesh N (2014) Closed talar dislocation without associated fracture a very rare injury, a case report. Journal of orthopaedic case reports 4: 10.

9. Haverkort J, Leenen L, van Wessem K (2015) Diagnosis and treatment of talar dislocation fractures illustrated by 3 case reports and review of literature. Int J Surg Case Rep 16: 106-111.

10. Dulani R, Shrivastava S, Dwidmuthe S, Purohit R (2012) Closed total (pantalar) dislocation of the talus with delayed presentation: a rare case repor and review of the literature. Ulus Travma Acil Cerrahi Derg 18: 268-270.

11. Ritsema GH (1988) Total talar dislocation. J Trauma 28: 692-694.

12. Taymaz A, Gunal I (2005) Complete dislocation of the talus unaccompanied by fracture. J Foot Ankle Surg 44: 156-158.

13. Hadji M, Golli M, Moalla R, Kmantar L, Hamdi A, et al. (2004) Conservative treatment of talar dislocation: a case report. Rev Chir Orthop Reparatrice Appar Mot 90: 285-288. 